\title{
Fast Approach to Evaluate MAP Reconstruction for Lesion Detection and Localization
}

\author{
Jinyi Qi and Ronald H Huesman \\ Lawrence Berkeley National Laboratory, One Cyclotron Road, Berkeley, CA 94720, USA
}

\begin{abstract}
Lesion detection is an important task in emission tomography. Localization ROC (LROC) studies are often used to analyze the lesion detection and localization performance. Most researchers rely on Monte Carlo reconstruction samples to obtain LROC curves, which can be very time-consuming for iterative algorithms. In this paper we develop a fast approach to obtain LROC curves that does not require Monte Carlo reconstructions. We use a channelized Hotelling observer model to search for lesions, and the results can be easily extended to other numerical observers. We theoretically analyzed the mean and covariance of the observer output. Assuming the observer outputs are multivariate Gaussian random variables, an LROC curve can be directly generated by integrating the conditional probability density functions. The high-dimensional integrals are calculated using a Monte Carlo method. The proposed approach is very fast because no iterative reconstruction is involved. Computer simulations show that the results of the proposed method match well with those obtained using the tradition LROC analysis.
\end{abstract}

\section{INTRODUCTION}

Iterative image reconstruction based on the maximum a posteriori (MAP) principle (also called penalized maximum likelihood) has been applied to emission tomography to deal with the low signal-to-noise ratio. ${ }^{1-4}$ Evaluation of the image quality for task performance is of importance for comparing different reconstruction methods and imaging systems. A standard methodology for studying lesion detectability is the receiver operating characteristic (ROC) study that compares true positive rate vs. false positive rate for human observers for the task of lesion detection. ${ }^{5-7}$ Building on the recent progress on the theoretical analysis of resolution and noise properties of iterative reconstruction algorithms ${ }^{8-18}$ and the development of numerical observer models, ${ }^{19,20}$ we have derived theoretical expressions for fast evaluation of the detectability of a random lesion in MAP reconstruction using ROC analysis. ${ }^{21}$ However, clinical tasks often involve the search for lesion location, which is not modeled in ROC analysis. A better approach to the evaluation of lesion detection may be to use the localization ROC (LROC) curve, ${ }^{22}$ which studies both detection and localization by separating the "true-positive" images into two mutually exclusive subsets at each rating criterion depending on whether or not the observer correctly identifies the lesion location. An LROC curve plots the joint proportion of true positive images with correctly located lesions as a function of false positive rate. Assuming the evaluation at a non-target location is unaffected by the presence or absence of a lesion, Swensson ${ }^{22}$ has derived a point-to-point relation between the ROC curve and the LROC curve. However, when the possible lesion locations are close to each other, this assumption is not valid.

Most researchers rely on Monte Carlo reconstruction samples to obtain LROC curves, which can be very time-consuming for iterative algorithms. In this paper we develop a fast approach to obtain LROC curves that does not require Monte Carlo reconstructions. We use a channelized Hotelling observer $(\mathrm{CHO})^{20}$ to search for lesions. The $\mathrm{CHO}$ is applied to all possible locations within a certain distance from the true target location. A lesion is correctly detected if the test statistic of the $\mathrm{CHO}$ achieves the maximum at the true target location (or within a certain preselected range) and exceeds the detection threshold. We analyzed the mean and covariance of the observer test statistics using theoretical approach that we have previously developed. Assuming the observer test statistics are multivariate Gaussian random variables, an LROC curve can be directly generated by integrating the conditional probability density functions. We calculate the high-dimensional integrals using a Monte Carlo sampling method. Since no iterative reconstruction is involved, the proposed method is very fast

Further author information: E-mail: \{jqi,rhhuesman\}@lbl.gov, Telephone: 15104864695 
compared with the traditional LROC approach. The results can be extended to other numerical observers, such as the channelized non-prewhitening observer used by Gifford and coworkers. ${ }^{23}$

This paper is different from a recent work presented by Khurd and Gindi, ${ }^{24}$ where similar numerical techniques were use to compute LROC curves. For fast computation, Khurd and Gindi ${ }^{24}$ assume not only that the evaluation at non-target locations is unaffected by the presence of a target, but also that the evaluations at different locations are independent. Therefore, their results do not work well when the noise correlation is too broad or possible lesion locations are too close. In comparison, we make no assumption of independence. We model the possible correlations between non-target locations, as well as the effect on the evaluation of non-target locations by the presence of the lesion. Hence the results presented here are more useful when possible lesion locations are close.

This paper is organized as follows. In Section 2 we review the theory of MAP reconstruction and numerical observers. Then we present the fast approach to calculate LROC curves in Section 3. Some derivations in our previous work ${ }^{15,21}$ are repeated here for completeness. In Section 4 we conduct computer-based Monte Carlo simulations to validate the theoretical results. The conclusions are drawn in Section 5.

\section{BACKGROUND}

\subsection{Data Model and MAP Reconstruction}

We model emission data as a collection of independent Poisson random variables with the (conditional) expectation, $\overline{\boldsymbol{y}} \in \mathbb{R}^{M \times 1}$, related to the unknown tracer distribution, $\boldsymbol{x} \in \mathbb{R}^{N \times 1}$, through an affine transform

$$
\overline{\boldsymbol{y}} \equiv E[\boldsymbol{y} \mid \boldsymbol{x}]=\boldsymbol{P} \boldsymbol{x}+\boldsymbol{r},
$$

where $E[\cdot \cdot \cdot]$ denotes conditional expectation, $\boldsymbol{P} \in \mathbb{R}^{M \times N}$ is the detection probability matrix with the $(i, j)$ th element equal to the probability of detecting an event from the $j$ th voxel at the $i$ th measurement with consideration of photon attenuation and detector efficiency, and $\boldsymbol{r} \in \mathbb{R}^{M \times 1}$ accounts for the presence of scattered and random events in the data.

The Poisson likelihood function is

$$
\mathrm{P}(\boldsymbol{y} \mid \boldsymbol{x})=\prod_{i} \frac{e^{-\bar{y}_{i}} \bar{y}_{i}^{y_{i}}}{y_{i} !}
$$

and the log likelihood function is given by

$$
L(\boldsymbol{y} \mid \boldsymbol{x})=\sum_{i}\left(y_{i} \log \bar{y}_{i}-\bar{y}_{i}-\log y_{i} !\right),
$$

where $\boldsymbol{y} \in \mathbb{R}^{M \times 1}$ are the measured sinogram data. For PET data that are precorrected for random events, a shifted-Poisson model ${ }^{25}$ can be used.

An ML estimate can be found by maximizing (3). A popular ML algorithm for PET reconstruction is the EM algorithm. ${ }^{26-28}$ However, ML solutions are very noisy because emission tomography is an ill-posed problem. Thus, some form of regularization (or prior function) is needed to reconstruct a reasonable image.

Bayesian methods regularize the image through the use of a prior distribution on the unknown image. Most image priors have a Gibbs distribution of the form

$$
\mathrm{P}(\boldsymbol{x})=\frac{1}{Z} e^{-\beta U(\boldsymbol{x})},
$$

where $U(\boldsymbol{x})$ is the energy function, $\beta$ is the smoothing parameter that controls the resolution of the reconstructed image, and $Z$ is a normalization constant. For log-quadratic priors that are studied here, the energy function can be expressed as

$$
U(\boldsymbol{x})=\frac{1}{2} \boldsymbol{x}^{\prime} \boldsymbol{R} \boldsymbol{x},
$$


where $\boldsymbol{R}$ is a positive definite (or semidefinite) matrix and ' denotes transpose. The commonly used pair-wise quadratic priors and thin plate priors ${ }^{29,30}$ are just special cases of (5).

Combining the likelihood function and the image prior, the MAP reconstruction is found as

$$
\hat{\boldsymbol{x}}(\boldsymbol{y})=\arg \max _{\boldsymbol{x} \geq 0}[L(\boldsymbol{y} \mid \boldsymbol{x})-\beta U(\boldsymbol{x})] .
$$

\subsection{Numerical Observers for LROC Analysis}

In an LROC study, observers are required to specify the most suspicious lesion location and a confidence rating for each image. To model this process, we march a numerical observer over each given image $\hat{\boldsymbol{x}}$. At each possible location $\boldsymbol{r}$, the numerical observer computes a test statistic, $\eta(\hat{\boldsymbol{x}}, \boldsymbol{r})$, by

$$
\eta(\hat{\boldsymbol{x}}, \boldsymbol{r})=\boldsymbol{t}^{\prime} \hat{\boldsymbol{x}}
$$

where $\boldsymbol{t}(\boldsymbol{r})$ is the observer template that depends on $\boldsymbol{r}$ and ' denotes transpose. The most suspicious location $\boldsymbol{r}^{*}(\hat{\boldsymbol{x}})$ and the rating $\eta^{*}(\hat{\boldsymbol{x}})$ are then obtained from

$$
\begin{aligned}
& \boldsymbol{r}^{*}(\hat{\boldsymbol{x}})=\arg \max _{\boldsymbol{r} \in \Omega} \eta(\hat{\boldsymbol{x}}, \boldsymbol{r}) \\
& \eta^{*}(\hat{\boldsymbol{x}})=\eta\left(\hat{\boldsymbol{x}}, \boldsymbol{r}^{*}\right)
\end{aligned}
$$

where $\Omega$ is the search region of the observer. This "maximum" detector is commonly used in detection tasks with location uncertainty. ${ }^{22-24}$

A decision whether there is a lesion present is made by comparing $\eta^{*}(\hat{\boldsymbol{x}})$ to a preselected threshold $\tau$. If $\eta^{*}(\hat{\boldsymbol{x}}) \geq \tau, \hat{\boldsymbol{x}}$ is determined to have a lesion; otherwise, it is not. Among the true positive cases, there are only a portion of images of which $\boldsymbol{r}^{*}(\hat{\boldsymbol{x}})$ is the correct lesion lesion, or more precisely, $\boldsymbol{r}^{*}(\hat{\boldsymbol{x}})$ is within a preselected tolerance region around the lesion. An LROC curve is obtained by plotting the joint fraction of the true positive images with correct localization vs. false positive fraction as we vary $\tau$. Compared to the conventional ROC curve, which always starts from $(0,0)$ and ends at $(1,1)$, an LROC curve starts from $(0,0)$, but ends at $\left(1, P_{C L}\right)$. $P_{C L}$, representing the probability of correct localization, is less than 1 in most cases, due to the localization error even with $100 \%$ false positive rate. As in ROC studies, the area under the LROC curve $\left(A_{L R O C}\right)$ is often used as a figure of merit.

We choose the channelized Hotelling observer ${ }^{19,31}$ because it is the most popular numerical observers used in ROC studies. Examples of other numerical observers in LROC studies can also be found in the literature. ${ }^{23,24}$ The test statistic of $\mathrm{CHO}$ at location $\boldsymbol{r}$ is

$$
\eta(\hat{\boldsymbol{x}}, \boldsymbol{r})=\boldsymbol{z}^{\prime} \boldsymbol{U}^{\prime} \boldsymbol{K}^{-1} \boldsymbol{U} \hat{\boldsymbol{x}}
$$

where $\boldsymbol{U} \in \mathbb{R}^{L \times M}$ represents $L$ frequency-selective channels that mimic the human visual system, $\boldsymbol{z} \equiv E\left[\hat{\boldsymbol{x}} \mid H_{1}\right]-$ $E\left[\hat{\boldsymbol{x}} \mid H_{0}\right]$, and $\boldsymbol{K} \in \mathbb{R}^{L \times L}$ is the covariance of the channel outputs, i.e.,

$$
\boldsymbol{K}=\frac{1}{2} \boldsymbol{U}\left(\Sigma_{\hat{\boldsymbol{x}} \mid H_{1}}+\Sigma_{\hat{\boldsymbol{x}} \mid H_{0}}\right) \boldsymbol{U}^{\prime}
$$

$H_{0}$ denotes lesion absent, $H_{1}$ denotes lesion present at location $\boldsymbol{r}, \Sigma_{\hat{\boldsymbol{x}} \mid H_{1}}$ and $\Sigma_{\hat{\boldsymbol{x}} \mid H_{0}}$ are the covariance matrices of $\hat{\boldsymbol{x}}$ under hypotheses of $H_{1}$ and $H_{0}$, respectively. Without loss of generality, here we assume that the probabilities of the two hypotheses are equal.

Note that $\boldsymbol{z}(\boldsymbol{r}), \boldsymbol{U}(\boldsymbol{r})$ and $\boldsymbol{K}(\boldsymbol{r})$ are all vector functions of $\boldsymbol{r}$. If the resolution and noise variance are stationary, the test statistics of the CHO for the whole image can be computed as a convolution. However, MAP reconstruction has spatially variant resolution and noise properties, so the observer template is spatially variant. Fortunately, empirical results have shown that the image properties of MAP reconstruction can be approximated to be locally stationary, ${ }^{14,15,18,32}$ so convolution can still be used in a local region. 


\section{FAST APPROACH TO COMPUTE THE LROC CURVE}

\subsection{Mean and Variance of the Test Statistics}

Since the test statistics $\eta(\hat{\boldsymbol{x}}, \boldsymbol{r})$ are results of a weighted sum of a number of random variables, we can assume that they follow a multivariate normal distribution. ${ }^{24}$ To generate the LROC curve, we need to the know the mean and variance of the test statistics, which can be written as

$$
\begin{aligned}
E\left[\eta(\hat{\boldsymbol{x}}, \boldsymbol{r}) \mid H_{k}\right] & =\boldsymbol{z}^{\prime} \boldsymbol{U}^{\prime} \boldsymbol{K}^{-1} \boldsymbol{U} E\left[\hat{\boldsymbol{x}} \mid H_{k}\right], \\
\Sigma_{\eta \mid H_{k}} & =\boldsymbol{T}^{\prime} \Sigma_{\hat{\boldsymbol{x}} \mid H_{k}} \boldsymbol{T},
\end{aligned}
$$

where the $j$ th column of $\boldsymbol{T}$ is the observer template at the $j$ th voxel, and $k=0,1$.

The central parts of the calculation are the expressions of $\boldsymbol{z}$ and covariance matrices $\Sigma_{\hat{\boldsymbol{x}} \mid H_{k}}$. Simplified expressions for fast computation of these quantities have been derived in our previous work. ${ }^{15,21}$ Here we briefly review the derivation. We focus on small lesions and low noise situations, so we use the first-order Taylor series expansion to approximate $\hat{\boldsymbol{x}}(\boldsymbol{y})$ around the point $\overline{\boldsymbol{y}} \equiv \boldsymbol{P} E\left[\boldsymbol{x} \mid H_{0}\right]+\boldsymbol{r}$, i.e.,

$$
\hat{\boldsymbol{x}}(\boldsymbol{y}) \approx \hat{\boldsymbol{x}}(\overline{\boldsymbol{y}})+\left.\nabla_{y} \hat{\boldsymbol{x}}(\boldsymbol{y})\right|_{\boldsymbol{y}=\overline{\boldsymbol{y}}}(\boldsymbol{y}-\overline{\boldsymbol{y}}) .
$$

The $(j, l)$ th element of $\nabla_{y} \hat{\boldsymbol{x}}(\boldsymbol{y})$ is $\partial \hat{\boldsymbol{x}}_{j}(\boldsymbol{y}) / \partial \boldsymbol{y}_{l}$. The expression of $\nabla_{y} \hat{\boldsymbol{x}}(\boldsymbol{y})$ was derived by Fessler ${ }^{12,13}$ using the fixed-point condition

$$
0=\left.\frac{\partial}{\partial x_{j}}[L(\boldsymbol{y} \mid \boldsymbol{x})-\beta U(\boldsymbol{x})]\right|_{\boldsymbol{x}=\hat{\boldsymbol{x}}(\boldsymbol{y})}, j=1, \ldots, M .
$$

Differentiating (15) with respect to $y_{i}$ by applying the chain rule and solving the resulting equation, we get

$$
\nabla_{y} \hat{\boldsymbol{x}}(\overline{\boldsymbol{y}}) \approx[\boldsymbol{F}+\beta \boldsymbol{R}]^{-1} \boldsymbol{P}^{\prime} \operatorname{diag}\left[\frac{1}{\bar{y}_{i}}\right]
$$

where $\boldsymbol{F}=\boldsymbol{P}^{\prime} \operatorname{diag}\left[\frac{1}{\bar{y}_{i}}\right] \boldsymbol{P}$ is the Fisher information matrix, and $\operatorname{diag}\left[y_{i}\right]$ denotes a diagonal matrix with the $(i, i)$ th element equal to $y_{i}$.

Using the above first-order Taylor series approximation, we can approximate $\boldsymbol{z}$ as

$$
\boldsymbol{z} \approx \nabla_{y} \hat{\boldsymbol{x}}(\overline{\boldsymbol{y}}) \boldsymbol{P} \overline{\boldsymbol{f}}_{l} \approx[\boldsymbol{F}+\beta \boldsymbol{R}]^{-1} \boldsymbol{F} \overline{\boldsymbol{f}}_{l},
$$

where $\overline{\boldsymbol{f}}_{l} \equiv E\left[\boldsymbol{x} \mid H_{1}\right]-E\left[\boldsymbol{x} \mid H_{0}\right]$ is the expectation of the lesion profile. Similarly, the covariance of MAP reconstruction can be approximated by

$$
\Sigma_{\hat{\boldsymbol{x}} \mid H_{k}} \approx[\boldsymbol{F}+\beta \boldsymbol{R}]^{-1} \boldsymbol{P}^{\prime} \operatorname{diag}\left[\frac{1}{\bar{y}_{i}}\right] \Sigma_{y \mid H_{k}} \operatorname{diag}\left[\frac{1}{\bar{y}_{i}}\right] \boldsymbol{P}[\boldsymbol{F}+\beta \boldsymbol{R}]^{-1} .
$$

where $\Sigma_{y \mid H_{k}}$ is the covariance matrix of the measurement $\boldsymbol{y}$ under $H_{k}, k=0,1$.

Given $\boldsymbol{x}, \boldsymbol{y}$ consists of independent Poisson random variables, of which the covariance is $\Sigma_{y \mid x}=\operatorname{diag}\left[\bar{y}_{i}\right]$ with $\overline{\boldsymbol{y}}=\boldsymbol{P} \boldsymbol{x}+\boldsymbol{r}$. When considering object variation, the overall covariance of the measurements is

$$
\Sigma_{y \mid H_{k}}=E\left\{\Sigma_{y \mid x} \mid H_{k}\right\}+\boldsymbol{P} \Sigma_{x \mid H_{k}} \boldsymbol{P}^{\prime},
$$

where $\Sigma_{x \mid H_{k}}$ is the covariance of the object variation under $H_{k}$.

For small lesions, we can assume that the presence of a lesion has almost no effect on the Poisson noise in the data, i.e., $E\left\{\Sigma_{y \mid x} \mid H_{k}\right\} \approx \operatorname{diag}\left[\bar{y}_{i}\right], k=0,1$. Therefore, we have

$$
\Sigma_{\hat{\boldsymbol{x}} \mid H_{k}} \approx[\boldsymbol{F}+\beta \boldsymbol{R}]^{-1}\left[\boldsymbol{F}+\boldsymbol{F} \Sigma_{x \mid H_{k}} \boldsymbol{F}\right][\boldsymbol{F}+\beta \boldsymbol{R}]^{-1} .
$$


Direct computation of (17) and (20) is very time-consuming due to the inverse of a large matrix $\boldsymbol{F}+\beta \boldsymbol{R}$. Assuming the image properties are locally stationary, we have obtained the following simplified expressions for fast computation ${ }^{15,21}$

$$
\begin{aligned}
\boldsymbol{z} & \approx \boldsymbol{Q}^{\prime} \operatorname{diag}\left[\frac{\lambda_{i}}{\lambda_{i}+\beta \mu_{i}}\right] \boldsymbol{Q} \overline{\boldsymbol{f}}_{l} \\
\Sigma_{\hat{\boldsymbol{x}} \mid H_{k}} \boldsymbol{e}_{l} & \approx \boldsymbol{Q}^{\prime} \operatorname{diag}\left[\frac{\lambda_{i}}{\left(\lambda_{i}+\beta \mu_{i}\right)^{2}}\right] \boldsymbol{Q} \boldsymbol{e}_{l}+\boldsymbol{Q}^{\prime} \operatorname{diag}\left[\frac{\lambda_{i}}{\lambda_{i}+\beta \mu_{i}}\right] \boldsymbol{Q} \Sigma_{x \mid H_{k}} \boldsymbol{Q}^{\prime} \operatorname{diag}\left[\frac{\lambda_{i}}{\lambda_{i}+\beta \mu_{i}}\right] \boldsymbol{Q} \boldsymbol{e}_{l},
\end{aligned}
$$

where $\left\{\lambda_{i}, i=1, \ldots, N\right\}$ and $\left\{\mu_{i}, i=1, \ldots, N\right\}$ are the Fourier coefficients of the column vectors corresponding to the lesion location of $\boldsymbol{F}$ and $\boldsymbol{R}$, respectively, $\boldsymbol{e}_{l}$ is a unit vector with 1 at the lesion location and zero elsewhere, and $\boldsymbol{Q}$ represents the two-dimensional (or three dimensional) Kronecker form of the Fourier transform. Details on the computation of $\boldsymbol{\lambda}$ and $\boldsymbol{\mu}$ can be found in our previous work. ${ }^{15}$ Note that $\lambda_{i}$ and $\mu_{i}$ change as we change the lesion location and the use of $\boldsymbol{e}_{l}$ indicates the approximation is on a column-by-column basis.

For further reduce the computational cost, we assume that $\lambda_{i}$ and $\mu_{i}$ are fixed within a local region, so the channel covariance can be approximated by

$$
\boldsymbol{K} \approx \tilde{\boldsymbol{U}} \operatorname{diag}\left[\frac{\lambda_{i}}{\left(\lambda_{i}+\beta \mu_{i}\right)^{2}}\right] \tilde{\boldsymbol{U}}^{\prime}+\frac{1}{2}\left(\boldsymbol{Q}^{\prime} \operatorname{diag}\left[\frac{\lambda_{i}}{\lambda_{i}+\beta \mu_{i}}\right] \tilde{\boldsymbol{U}}^{\prime}\right)^{\prime} \boldsymbol{U}\left(\Sigma_{\boldsymbol{x} \mid H_{1}}+\Sigma \boldsymbol{x}_{\mid H_{0}}\right)\left(\boldsymbol{Q}^{\prime} \operatorname{diag}\left[\frac{\lambda_{i}}{\lambda_{i}+\beta \mu_{i}}\right] \tilde{\boldsymbol{U}}^{\prime}\right)
$$

where $\tilde{\boldsymbol{U}}=\boldsymbol{U} \boldsymbol{Q}^{\prime}$ are the Fourier coefficients of the channel functions. Substituting (21) and (23) into (10), we get the expression of the observer template

$$
\boldsymbol{t}_{C H O} \approx \boldsymbol{U}^{\prime} \boldsymbol{K}^{-1} \tilde{\boldsymbol{U}} \operatorname{diag}\left[\frac{\lambda_{i}}{\lambda_{i}+\beta \mu_{i}}\right] \boldsymbol{Q} \overline{\boldsymbol{f}}_{l} .
$$

Within the local region, we can use convolution to compute $\eta(\hat{\boldsymbol{x}}, \boldsymbol{r})$ as

$$
\eta(\hat{\boldsymbol{x}}, \boldsymbol{r}) \approx \boldsymbol{t}_{C H O} * \hat{\boldsymbol{x}}
$$

where $*$ denotes two- (or three-) dimensional convolution although we write images as vectors.

In most cases, we are looking for a possible lesion in an otherwise uniform background, at least within a local region. We can approximate $E\left[\eta \mid H_{0}\right] \approx 0$ because humans do not use DC information to make the decision. This is equivalent to the subtraction of the background used by others. ${ }^{23,24}$ As a result,

$$
E\left[\eta(\hat{\boldsymbol{x}}, \boldsymbol{r}) \mid H_{1}\right] \approx \boldsymbol{t}_{C H O} * \boldsymbol{z} .
$$

Thus the mean and covariance of the test statistics can be readily computed using the Fourier transform.

\subsection{The LROC Curve}

Once we have the mean and covariance of $\eta(\hat{\boldsymbol{x}}, \boldsymbol{r})$, we can directly generate Monte Carlo samples of $\eta(\hat{\boldsymbol{x}}, \boldsymbol{r})$, from which we can plot LROC curves. The advantage of this approach is that it does not require Monte Carlo reconstructions, so it dramatically reduces the computational time for LROC studies. A Monte Carlo sample is generated by filtering a white noise field (zero mean and unit variance) in the frequency domain and adding an appropriate mean. The maximum and the corresponding location are found in each Monte Carlo sample. They are compared to a threshold and the true lesion location to calculate the true positive rate and false positive rate. As we vary the threshold, the true positive rate and false positive rate will change. An LROC curve is then obtained by plotting the joint fraction of the true positive images with correct localization vs. the false positive rate.

As we mentioned before, a correct localization is determined by comparing distance between the most suspicious location and the true lesion site with a preselected tolerant range. Obviously the probability of correct localization depends on the tolerance range. As we increase the tolerance, the probability of correct localization increases. At the limit when the tolerance region covers the whole search region $\Omega$, the LROC curve overlaps with the ROC curve. Clearly Swensson's model, where there is a point-to-point relation between the ROC curve and the LROC curve, is not valid in this situation. The reason is because the possible lesion sites are very close and hence the independence assumption does not hold. 


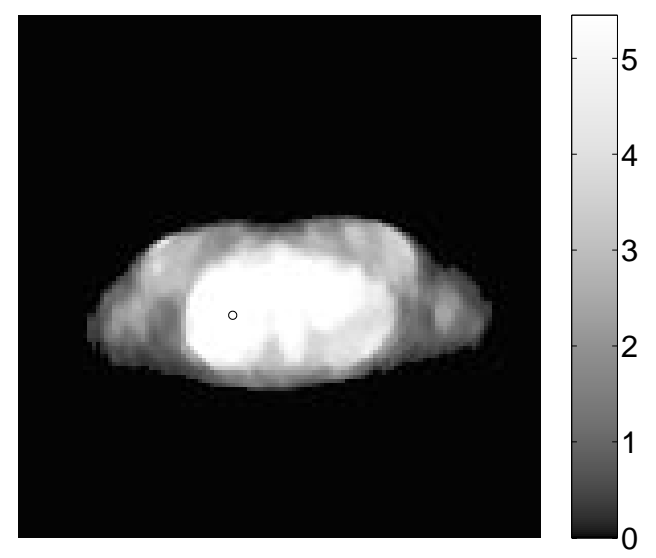

Figure 1. The background phantom image and the location of the simulated lesion.

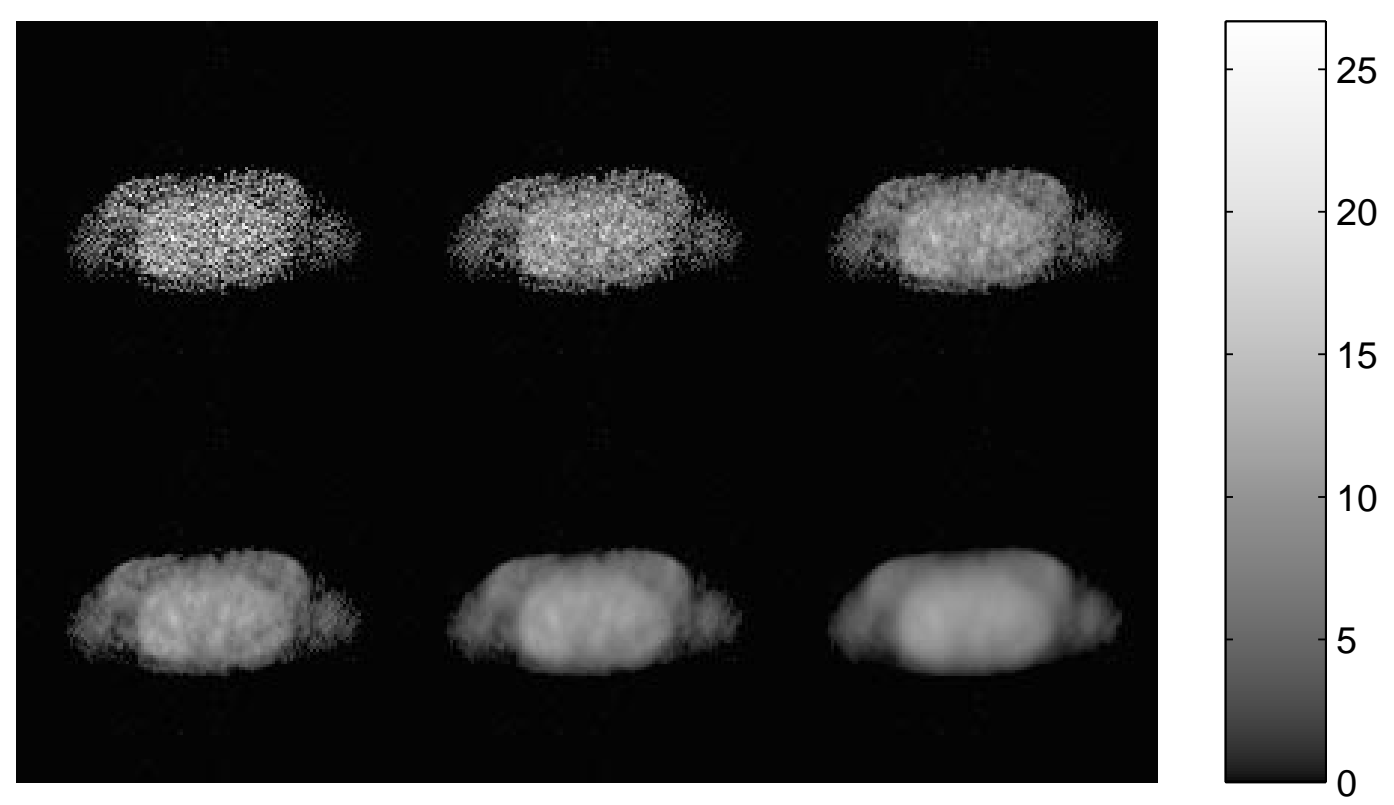

Figure 2. Reconstructed images of a noisy data set with a lesion. Top row, from left to right, $\beta=1,3,10 ;$ bottom row, from left to right, $\beta=30,100,300$.

\section{MONTE CARLO SIMULATIONS}

We conducted computer simulations to validate the proposed method. We simulated an ECAT HR+ whole-body PET scanner (CTI PET Systems, Knoxville, TN) operating in 2D mode. The sinogram data have 288 angles of view and 288 lines of response in each view. The background phantom image is shown in Figure 1. The lesion is 8 - $\mathrm{mm}$ in diameter. All images are represented by $128 \times 128$-mm square pixels.

For the traditional LROC study, we reconstructed 500 pair images (with and without a lesion) using a preconditioned conjugated gradient MAP algorithm ${ }^{33}$ with different smoothing parameters. The data were generated by forward projecting the phantom image with and without a lesion. Photon attenuation was modeled. Poisson noise was added to the sinogram data after scaling the expected total number of events to 200k. Examples of the reconstructed images are shown in Figure 2. We applied the CHO to the reconstructed images to obtain the observer test statistics. Two sets of channel functions were studied: (i) five rotationally symmetric, 


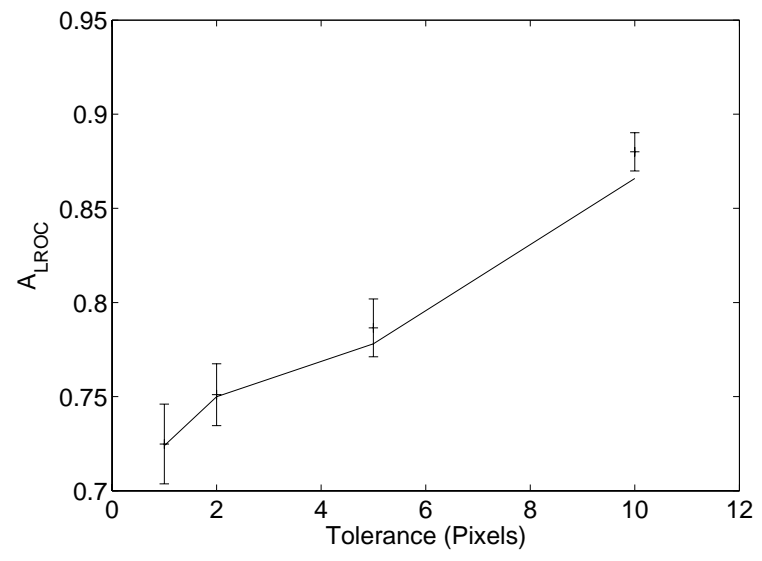

(a)

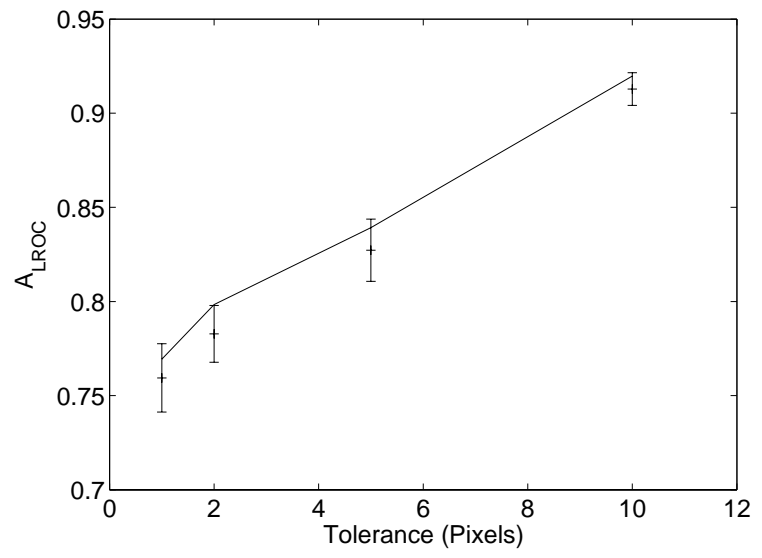

(b)

Figure 3. The area under the LROC curve as a function of the tolerance distance for correct localization. (a) DOG channels; (b) SQR channels. For all the points, the search radius is 10 pixels and $\beta=10$.

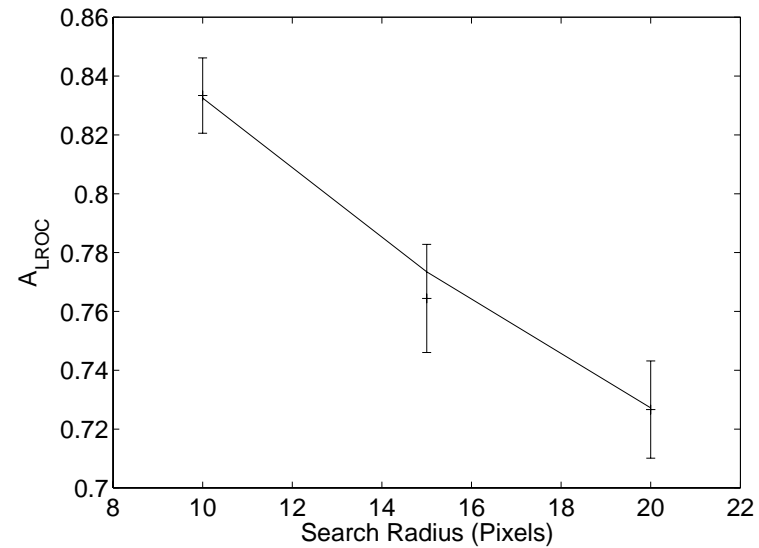

(a)

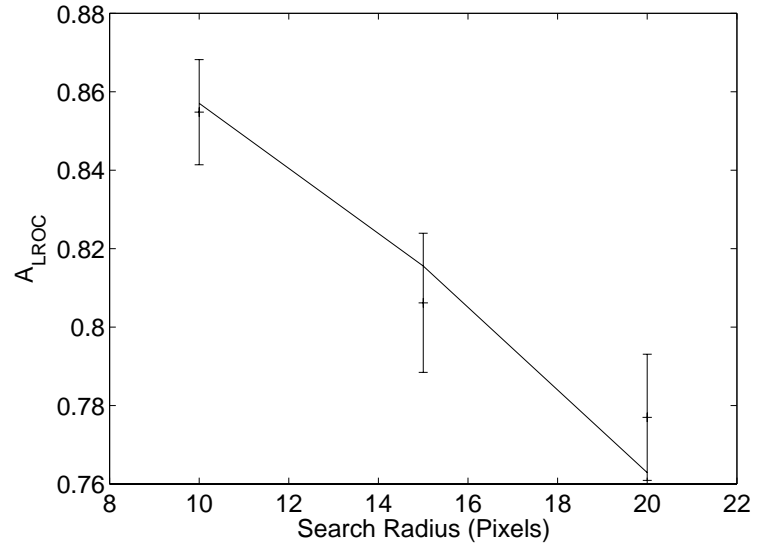

(b)

Figure 4. The area under the LROC curve as a function of the radius of the search region. (a) DOG channels; (b) SQR channels. For all the points, the tolerance distance is 5 pixels and $\beta=100$.

non-overlapping square channels (SQR); and (ii) three difference-of-Gaussian channels (DOG). These channel functions are similar to that used in others. ${ }^{31,34}$ For the proposed method, the observer test statistics were obtained by sampling the conditional probability density function directly. From the observer test statistics, LROC curves were generated by plotting the correctly identified lesion fraction versus the false positive fraction. The area under the LROC curve was integrated numerically.

Comparisons of $A_{L R O C}$ computed by the two methods are shown in Figures 3-5. The error bars were computed using a bootstrap method. Figure 3 plots $A_{L R O C}$ as a function of the tolerance distance for correct localization. As we expected, $A_{L R O C}$ increases as the tolerance distance increases. Figure 4 shows $A_{L R O C}$ decreases as we increase the search radius. This is expected because increasing the search radius will reduce the probability of correct localization in the true positive cases. Figure 5 plots $A_{L R O C}$ as a function of the prior parameter $\beta$. In most cases, the results of the propose method match those of the traditional LROC method reasonably well. However, we did find that the accuracy of the proposed method deteriorates as we increase the search radius or $\beta$. This is the limitation of the locally stationary assumption that is used in the fast computation. 


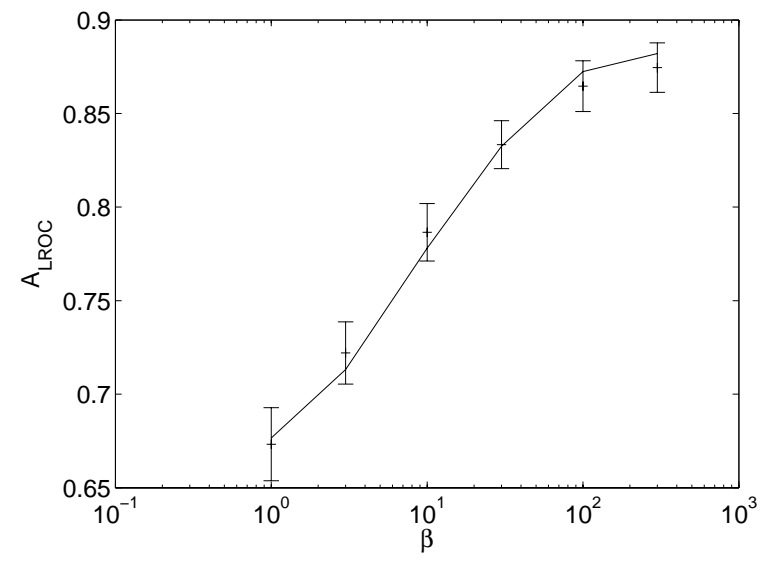

(a)

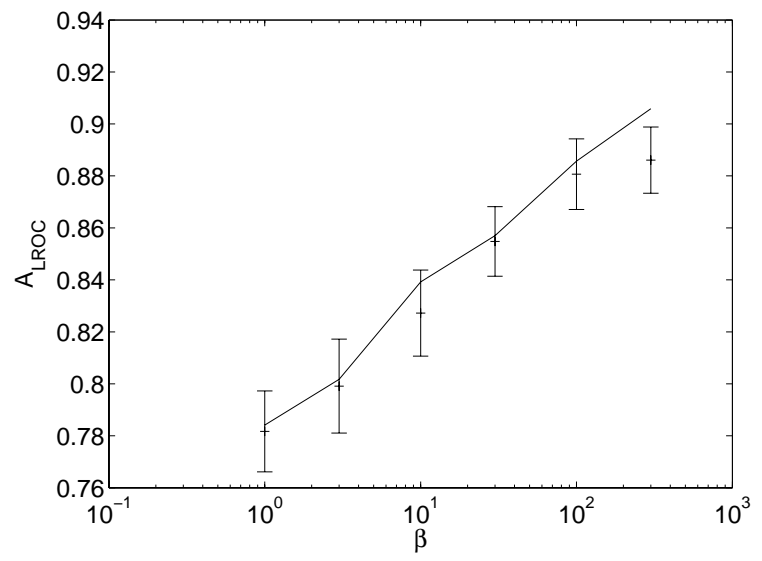

(b)

Figure 5. The area under the LROC curve as a function of the prior parameter $\beta$. (a) DOG channels; (b) SQR channels. For all the points, the tolerance distance is 5 pixels and the search radius is 10 pixels.

The advantage of the proposed method is that it does not require Monte Carlo reconstructions. The time to generate one LROC curve using the proposed method is about 82 seconds (using a Matlab code) with $\lambda_{i}$ and $\mu_{i}$ precomputed, while Monte Carlo reconstructions (using a c-code) used in the traditional LROC method (500 pairs for each smoothing parameter) took about 20 days on a Sun Ultra 60 workstation with $450-\mathrm{MHz}$ CPU. Therefore, the proposed method will allow fast evaluation of MAP reconstruction for a large number of parameters, heretofore, impractical with traditional methods.

\section{CONCLUSION}

We have developed a fast approach to evaluate image quality of MAP reconstruction for LROC studies. The method does not require Monte Carlo reconstruction samples and hence greatly reduces the computation time. Computer simulation results show that the accuracy of the proposed method is comparable to that of the traditional LROC method. The method currently is limited to a local region around the lesion. In future work we will develop techniques to eliminate this limitation.

\section{Acknowledgments}

The authors would like to thank Dr. H. H. Barrett for helpful discussions on this topic.

This work is supported in part by the National Institute of Biomedical Imaging and Bioengineering under grant nos. R01 EB00194, R01 EB00363, and by the Director, Office of Science, Office of Biological and Environmental Research, Medical Sciences Division, of the U.S. Department of Energy under contract no. DEAC03-76SF00098.

\section{REFERENCES}

1. J. A. Fessler, "Penalized weighted least-squares image reconstruction for PET," IEEE Transactions on Medical Imaging 13, pp. 290-300, June 1994.

2. E. Mumcuoglu, R. Leahy, S. Cherry, and Z. Zhou, "Fast gradient-based methods for Bayesian reconstruction of transmission and emission PET images," IEEE Transactions on Medical Imaging 13, pp. 687-701, December 1994.

3. J. A. Fessler and A. O. Hero, "Penalized maximum-likelihood image reconstruction using space-alternating generalized EM algorithms," IEEE Transactions on Image Processing 4, pp. 1417-1429, Oct 1995.

4. C. Bouman and K. Sauer, "A unified approach to statistical tomography using coordinate descent optimization," IEEE Transactions on Image Processing 5, pp. 480-492, March 1996. 
5. D. R. Gilland, B. M. W. Tsui, C. E. Metz, R. J. Jaszczak, and J. R. Perry, "An evaluation of maximum likelihood-expectation maximization reconstruction for SPECT by ROC analysis," The Journal of Nuclear Medicine 33, pp. 451-457, 1992.

6. J. Llacer, "Results of a clinical receiver operating characteristic study comparing filtered backprojection and maximum likelihood estimator images in FDG PET studies," The Journal of Nuclear Medicine 34(7), pp. 1198-1203, 1993.

7. T. H. Farquhar, Improved Lesion Detection in Whole Body PET. PhD thesis, University of California, Los Angeles, 1998.

8. H. H. Barrett, D. W. Wilson, and B. M. W. Tsui, "Noise properties of the EM algorithm: I. theory," Physics in Medicine and Biology 39, pp. 833-846, 1994.

9. W. Wang and G. Gindi, "Noise analysis of MAP-EM algorithms for emission tomography," Physics in Medicine and Biology 42, pp. 2215-2232, 1997.

10. E. J. Soares, C. Byrne, and S. Glick, "Noise characterization of block-iterative reconstruction algorithms: 1. theory," IEEE Transactions on Medical Imaging 19, pp. 261-270, April 2000.

11. J. Qi, "A unified noise analysis for iterative image estimation," Physics in Medicine and Biology 48, pp. 3505-3519, 2003.

12. J. Fessler, "Mean and variance of implicitly defined biased estimators (such as penalized maximum likelihood): Applications to tomography," IEEE Transactions on Image Processing 5, pp. 493-506, March 1996.

13. J. A. Fessler and W. L. Rogers, "Spatial resolution properties of penalized-likelihood image reconstruction: Spatial-invariant tomographs," IEEE Transactions on Image Processing 9, pp. 1346-1358, September 1996.

14. J. Qi and R. M. Leahy, "A theoretical study of the contrast recovery and variance of MAP reconstructions from PET data," IEEE Transactions on Medical Imaging 18, pp. 293-305, April 1999.

15. J. Qi and R. M. Leahy, "Resolution and noise properties of MAP reconstruction for fully 3D PET," IEEE Transactions on Medical Imaging 19, pp. 493-506, May 2000.

16. C. K. Abbey and H. H. Barrett, "Observer signal-to-noise ratios for the ML-EM algorithm," in Proceedings of SPIE, 2712, pp. 47-58, 1996.

17. J. A. Fessler and A. Yendiki, "Channelized Hotelling observer performance for penalized-likelihood image reconstruction," in Proceedings of IEEE Nuclear Science Symposium and Medical Imaging Conference, pp. 1040-1044, 2002.

18. Y. Xing, I. T. Hsiao, and G. Gindi, "Rapid calculation of detectability in Bayesian single photon emission computed tomography," Physics in Medicine and Biology 48, pp. 3755-3773, 2003.

19. J. Yao and H. H. Barrett, "Predicting human performance by a channelized Hotelling model," in SPIE Mathematical Methods in Medical Imaging, 1768, pp. 161-168, 1992.

20. H. H. Barrett, J. Yao, J. Rolland, and K. Myers, "Model observers for assessment of image quality," Proc. Natl. Acad. Sci. 90, pp. 9758-9765, 1993.

21. J. Qi, "Analysis of lesion detectability in Bayesian emission reconstruction with non-stationary object variability," IEEE Transactions on Medical Imaging, 2004, to appear.

22. R. G. Swensson, "Unified measurement of observer performance in detecting and localizing target objects on images," Medical Physics 23, pp. 1709-1725, 1996.

23. H. C. Gifford, P. H. Pretorius, and M. A. King, "Comparison of human- and model-observer LROC studies," in Proceedings of SPIE, 5034, pp. 112-122, 2003.

24. P. Khurd and G. Gindi, "Rapid computation of LROC figures of merit using numerical observers (for SPECT/PET reconstruction)," in Proceedings of IEEE Nuclear Science Symposium and Medical Imaging Conference, pp. M9-3, 2003.

25. M. Yavuz and J. A. Fessler, "Statistical image reconstruction methods for randoms-precorrected PET scans," Medical Image Analysis 2(4), pp. 369-378, 1998.

26. A. Dempster, N. Laird, and D. Rubin, "Maximum likelihood from incomplete data via the EM algorithm," Journal of Royal Statistical Society, Series B 39(1), pp. 1-38, 1977.

27. L. Shepp and Y. Vardi, "Maximum likelihood reconstruction for emission tomography," IEEE Transactions on Medical Imaging 1, pp. 113-122, October 1982. 
28. K. Lange and R. Carson, "EM reconstruction algorithms for emission and transmission tomography," Journal of Computer Assisted Tomography 8, pp. 306-316, April 1984.

29. A. Blake and A. Zisserman, Visual Reconstruction, The MIT Press, 1987.

30. S.-J. Lee, A. Rangarajan, and G. Gindi, "Bayesian image reconstruction in SPECT using higher order mechanical models as priors," IEEE Transactions on Medical Imaging 14, pp. 669-680, December 1995.

31. K. J. Myers and H. H. Barrett, "Addition of a channel mechanism to the ideal-observer model," Journal of the Optical Society of America A 4(12), pp. 2447-2457, 1987.

32. J. W. Stayman and J. A. Fessler, "Regularization for uniform spatial resolution properties in penalizedlikelihood image reconstruction," IEEE Transactions on Medical Imaging 19, pp. 601-615, June 2000.

33. J. Qi, R. M. Leahy, S. R. Cherry, A. Chatziioannou, and T. H. Farquhar, "High resolution 3D Bayesian image reconstruction using the microPET small animal scanner," Physics in Medicine and Biology 43(4), pp. 1001-1013, 1998.

34. M. King, D. de Vries, and E. Soares, "Comparison of the channelized Hotelling and human observers for lesion detection in hepatic SPECT imaging," in Proceedings of SPIE, 3036, pp. 14-20, 1997. 